\title{
Atopy Is Inversely Related to Schistosome Infection Intensity: A Comparative Study in Zimbabwean Villages with Distinct Levels of Schistosoma haematobium Infection
}

\author{
Nadine Rujeni ${ }^{\mathrm{a}}$ Norman Nausch ${ }^{\mathrm{a}}$ Claire D. Bourke ${ }^{\mathrm{a}}$ Nicholas Midzi $^{\mathrm{c}}$ \\ Takafira Mduluza $^{d}$ David W. Taylor ${ }^{\text {b }}$ Francisca Mutapi ${ }^{a}$ \\ a Institute of Immunology and Infection Research, and ${ }^{\mathrm{b}}$ Centre for Infectious Diseases, School of Biological \\ Sciences, University of Edinburgh, Ashworth Laboratories, Edinburgh, UK; ' ${ }^{C}$ ational Institutes of Health \\ Research, and ${ }^{\mathrm{d}}$ Department of Biochemistry, University of Zimbabwe, Harare, Zimbabwe
}

\section{Key Words}

Atopy · Immunoglobulins E and G4 · Schistosoma

haematobium infection - Dermatophagoides pteronyssinus . Antibody

\begin{abstract}
Background: The hygiene hypothesis suggests that parasitic infections protect against allergic diseases by modulating the host's immune responses. Experimental studies indicate that this protection depends on the intensity of parasitic infection, but this observation has not been tested in human populations. The aim of this study is to investigate whether the intensity of Schistosoma haematobium infection is related to atopic responses and whether this relationship differs between populations with distinct parasite transmission dynamics. Methods: The study was conducted in two villages with different Schistosoma haematobium transmission dynamics, i.e. high $(n=365)$ and low $(n=307)$ transmission. Allergic reactivity to the common house dust mite (Dermatophagoides pteronyssinus) was measured by skin prick tests and allergen-specific $\lg \mathrm{E}$ and $\lg \mathrm{G} 4$ quantified by enzymelinked immunosorbent assay. Atopic responses were related to current infection intensity and schistosome transmission levels. Results: Schistosome infection intensity was nega-
\end{abstract}

\section{KARGER}

E-Mail karger@karger.ch www.karger.com/iaa

\section{(0) 2012 S. Karger AG, Basel \\ $1018-2438 / 12 / 1583-0288 \$ 38.00 / 0$}

Karger

Open access

This is an Open Access article licensed under the terms of the Creative Commons Attribution-NonCommercial 3.0 License (www.karger.com/OA-license-WT), applicable to the online version of the article only. Distribution for noncommercial purposes only. tively associated with the skin prick reactivity, mite-specific $\operatorname{lgE}$ and the ratio $\operatorname{lgE} / \operatorname{lgG} 4$ in the high-transmission village. However, when only low levels of infection were analyzed in the 2 villages, there was no correlation between mite-specific responses and infection intensity. Conclusion: The relationship between schistosome infection and atopic responses is dependent on the intensity of current schistosome infection. Thus, consistent with results from animal models, with an increasing parasite burden, the immunoregulation of immune responses to allergens appears to become more pronounced.

Copyright $\odot 2012$ S. Karger AG, Basel

\section{Introduction}

Atopy, i.e. the genetic predisposition to become IgEsensitized to environmental allergens [1], is an important risk factor for allergic diseases but only a small proportion of atopic individuals develop clinical symptoms $[2,3]$. The clinical manifestation of allergic symptoms in atopic individuals is influenced by environmental factors such as air pollution, diet and exposure to pathogens [4], leading to the heterogeneous distribution of atopic diseases worldwide [5]. The rise in atopy with urbanization 
has been attributed to reduced exposure to pathogens and their products in childhood as a result of improved hygiene [4]. In particular, helminth parasites are believed to induce regulatory immune responses that dampen atopic responses [6], but this remains controversial [7, 8]. Animal studies have shown that only heavy, chronic helminth infection provides protection against atopy [9], which would explain some of the conflicting results from human studies $[8,10]$. To date, despite the several elegant mechanistic studies in the mouse models of the relationship between helminth infections and atopy [11], only a few of the hypotheses and mechanisms have been validated in human studies. In particular, there is a paucity of studies investigating the effects of heterogeneities in host and parasite genotype, parasite transmission dynamics and previous history of host infection.

A meta-analysis of human epidemiological studies has suggested that in areas of high helminth (geohelminth) prevalence, the risk of allergy is decreased, whereas in areas of low prevalence this risk is increased [12]. This highlights the importance of helminth infection levels in the epidemiological patterns of helminth and atopy in human populations.

We therefore set out to investigate the effect of different parasite transmission dynamics on atopic responses in human populations naturally exposed to Schistosoma haematobium which causes urogenital schistosomiasis. To capture the dynamics of the relationship between helminth infection and atopy, the study was conducted in two villages of differing schistosome transmission dynamics, i.e. low and high transmission areas, resulting in differences in current infection intensity as well as in the cumulative history of schistosome infection $[13,14]$. Skin prick reactivity to the house dust mite (HDM) (Dermatophagoides pteronyssinus) - the most prevalent allergen in Zimbabwe $[15,16]$ - as well as levels of schistosomespecific and HDM-specific IgE and IgG4 antibody responses were related to schistosome infection intensity in the two villages.

\section{Materials and Methods}

\section{Study Design}

The study was comparative, contrasting the levels of atopic responses in high- and low-schistosome infection villages. This comparative approach, used by us and in other studies [14, 17-20], is very powerful for studying disease and immunology patterns where the history of exposure to the pathogen cannot be separated from age in lifelong residents of endemic areas. As previously published [21], Zimbabwe has low levels of geohelminths. In addition, Schistosoma mansoni levels are also low in most regions of Zimbabwe [22], while S. haematobium is the most prevalent helminth infection in Zimbabwe. In this study area, the prevalence of S. mansoni was $2 \%$, thus the study focused on S. haematobium infection. Differences in infection levels reflect the differences in infection transmission rates and in the history of infection [13]. Subjects in the high-infection village accumulated infection more rapidly, acquiring higher infection intensities at a younger age than their counterparts in the low-infection village [13].

\section{Study Area and Population}

The study was conducted in two villages, Magaya and Chitate, in the Mashonaland East province of Zimbabwe where S. haematobium is endemic. The study villages are in close proximity within a $10-\mathrm{km}$ range of each other and villagers are of similar ethnicity (Shona) and socioeconomic background (rural subsistence farmers). Safe water and sanitation coverage are equally poor in the villages (indicated by our questionnaire studies). The only obvious difference in the villages is the seasonality of the rivers which provide habitats for schistosome intermediate-host snails.

Magaya village is characterized by perennial rivers which lead to high transmission rates of schistosome parasites compared to Chitate village which is characterized by seasonal streams. In addition, households in Magaya are built along the rivers, whereas in Chitate they are more dispersed and are built further from the rivers. Human contact with water harboring cercariae, the infective stage of schistosomes, is frequent (assessed by questionnaire studies) in this area due to insufficient safe water and sanitation facilities.

In 2002, a revised stratification based on a national parasite prevalence survey, Health Management Information Systems data, entomological data and expert opinion classified our study area under the sporadic transmission regions with low transmission $[23,24]$ meaning that this is a mesoendemic area for Plasmodium transmission and malaria [25].

\section{Ethical Statement}

Permission to conduct the study was obtained from the Provincial Medical Director, institutional and ethical approval was received from the University of Zimbabwe and the Medical Research Council of Zimbabwe, respectively. All participants had the aims and procedures of the project explained and written consent was obtained before enrolment into the study, and all were offered treatment with the recommended dose $(40 \mathrm{mg} / \mathrm{kg}$ of body weight) of the antihelminthic drug, praziquantel. For young children, written consent was obtained from parents/guardians.

\section{Sample Collection}

The gold standard method for detecting and quantifying schistosome infection is through microscopic enumeration of eggs excreted in stool or urine [26]. Stool samples for the diagnosis of $S$. mansoni and geohelminths and urine specimens for the diagnosis of $S$. haematobium infection were collected on 3 consecutive days and processed using standard procedures [23, 24]. For infants unable to produce stool or urine samples on demand, an overnight sample was collected by the parent/guardian and submitted on the following day. Up to $5 \mathrm{ml}$ venous blood was collected from each participant in citrate tubes, which was used to obtain sera for antibody assays. Malaria status was determined by 
blood smears and confirmed by a serological Paracheck dipstick (Orchid Biomedical systems).

\section{Inclusion Criteria}

To be included in the study, participants had to (1) be lifelong residents of the village (assessed by questionnaire), (2) be free from the geohelminth, $S$. mansoni, and Plasmodium infections (7 people were excluded on this criterion because they were $S$. mansoni-positive but none was positive for Plasmodium infection, which is consistent with this area being mesondemic for Plasmodium transmission [25]), (3) not have previously received antihelminthic treatment (no one was excluded on this criterion), (4) provide urine and stool samples for helminth diagnosis and a blood sample for serological assays (107 people were excluded on this criterion because they did not provide either of the samples, see table 1a). A total of 672 individuals fulfilled the inclusion criteria and formed the study populations of Magaya ( $n=365$, age range $3-86$ years) and Chitate ( $\mathrm{n}=307$, age range $1-86$ years) described in table la.

\section{Skin Prick Test}

Skin prick tests (SPTs) were conducted (on a small consenting population, $\mathrm{n}=332$ ) using 4 allergens prevalent in Zimbabwe [15]: HDM, Grass mix, Alternaria tenius and Aspergillus fumigatus. Briefly, a drop of each allergen was placed on the upper side of the lower arm before the skin beneath the drop was pricked using a lancet. The skin was then wiped, and 15 min later, the size of the wheal diameter was measured whenever the skin reacted for a given allergen. Histamine and saline solutions (Diagenics Ltd) were used as positive and negative controls, respectively. Reactions were considered positive if the wheal diameter was equal to or greater than the positive control.

This procedure showed that allergy to the HDM was the most prevalent (67.8\% of the positive reactions were due to HDM alone), consistent with previous studies from Zimbabwe $[15,16]$. Therefore, serological assays focused on D. pteronyssinus-specific responses.

\section{Serology and Laboratory Assays}

Levels of antibodies directed against parasite antigens were measured by indirect enzyme-linked immunosorbent assay (ELISA) following published procedures [13] using soluble S. haematobium adult-worm antigen (SWAP) and soluble egg antigen (SEA) from the Theodor Bilharz Institute (Egypt). The parasite strain is one used in previous epidemiological studies [25, 26]. Mite-specific responses were measured by ELISA using natural affinity-purified $D$. pteronyssinus Der $\mathrm{p} 1$ allergen from Indoor Biotechnologies. Microtiter plates were coated overnight at $4^{\circ} \mathrm{C}$ with $50 \mu \mathrm{l} /$ well of Der p 1 allergen in $5 \mu \mathrm{g} / \mathrm{ml}$ carbonate-bicarbonate buffer. Serum samples were diluted at 1:10 and the secondary antibodies, anti-human horseradish peroxide conjugated IgE (Sigma) and IgG4 (the binding site) at 1:1,000. The substrate 2, 2'azino-bis-(3-benzthiazoline-6-sulfonic acid) (Southern Biotech) was used for the colorimetric reaction and the optical density read at $405 \mathrm{~nm}$. All sera were assayed in duplicate and a pool of sera from 6 individuals presenting with the highest skin-prick reactivity was included on each plate to serve as a positive control. A blank control containing no sera was included on each plate (in duplicate) and the background absorbance of reagents in the absence of serum was subtracted from all readings.

\section{Statistical Analysis}

Schistosome infection prevalence and intensity were compared between the 2 villages by Pearson $\chi^{2}$ test and analysis of variance (ANOVA), respectively. Age was categorized into 5 groups (table 1). The ANOVA used sequential sums of squares to calculate the mean squares to derive the test statistic. These allow the hierarchical decomposition of the sources of variation in the dependent variable, so that the effects of the preceding variable on the test variable are taken into account before testing for the variable of interest [19]. In this way, they allow for confounding variables before testing for the effects of the variable of interest. This approach has been used to test for the relationship between schistosome infection and immune responses or other markers of immunity or pathology, while allowing for the potential confounding effects of the sex and age of the host [27].

The effect of mite-specific antibodies on the SPT was assessed by ANOVA using sequential sums of squares to account for the effects of host age, sex, village of residence and infection intensity [28].

A binary stepwise logistic regression was used to determine the relationship between SPT status and antibody levels with age, sex, village of residence and infection intensity included in the model.

Following exploratory plots, the hypothesis that helminth infection is negatively associated with atopy was tested using a 1-tailed Pearson correlation analysis between the SPT results and infection intensity while controlling for the effects of host age, sex and village of residence. To test whether this association occurs at the immunological level, the same correlation analysis was conducted relating antibody levels to infection intensity in each village. The effect of infection intensity on antibody responses (Der p 1- and schistosome-specific) was determined by multivariate analysis using sequential sums of squares, entering the variable 'infection intensity' last in the model [28]. $\beta$-coefficients (for the direction of infection-antibody relationship) were calculated using linear regression analysis on residuals saved after accounting for the variations due to age, sex and village. All statistical analyses were conducted using PASW statistics (version 17; formerly called SPSS) and values of $\mathrm{p}<0.05$ were considered significant.

\section{Results}

\section{S. haematobium Infection Levels Differ Significantly between the Two Villages}

Schistosome infection levels were significantly higher in Magaya than Chitate with a prevalence of 45.4 versus $8.5 \%\left(\chi^{2}=111.4\right.$, d.f. $\left.=1, \mathrm{p}<0.001\right)$ and mean infection intensity of $26.3 \mathrm{eggs} / 10 \mathrm{ml}$ versus $0.755 \mathrm{eggs} / 10 \mathrm{ml}$ $\left(\mathrm{F}_{1,662}=92.75, \mathrm{p}<0.001\right)$, respectively. When partitioned by age, there were significant differences in infection levels in the two areas in younger age groups (fig. 1). Infection peaked at a higher level and at an earlier age in $\mathrm{Ma}-$ gaya (fig. 1), a phenomenon referred to as peak shift [29] and confirmed by a significant age and village interaction $\left(\mathrm{F}_{4,662}=5.938, \mathrm{p}<0.001\right)[13]$. 
Table 1. Study population description

a Demography and socioeconomic status in the study areas

\begin{tabular}{|c|c|c|}
\hline & Magaya (HTA) & Chitate (LTA) \\
\hline Number of participants (baseline) & 442 & 344 \\
\hline Mean age, years (range) & $11.4(3-86)$ & $18.5(1.5-86)$ \\
\hline Sex ratio $(M / F)$ & $0.825(199 / 241)$ & $0.57(127 / 221)$ \\
\hline Ethnic group & Shona & Shona \\
\hline Socioeconomic status & rural subsistence farmers & rural subsistence farmers \\
\hline S. haematobium prevalence & $52.94 \%(95 \%$ CI $48-57.67)$ & $8.6 \%(95 \%$ CI $5.9-12.1)$ \\
\hline S. haematobium intensity, eggs/10 $\mathrm{ml}$ (range) & $44.79(0-859)$, SEM $=5.654$ & $0.958(0-70.3), \mathrm{SEM}=0.2723$ \\
\hline S. mansoni cases (excluded) & 6 cases $(7-13$ years old $)$ & 1 case $(69$ years old $)$ \\
\hline Malaria prevalence & $0 \%$ & $0 \%$ \\
\hline STH prevalence & $0 \%$ & $0 \%$ \\
\hline No urine sample provided (excluded) & 16 cases $(3-26$ years old) & 2 cases (unknown age) \\
\hline \multicolumn{3}{|l|}{ No blood sample provided and/or } \\
\hline no age record (excluded) & 55 & 34 \\
\hline Total number of participants included & 365 & 307 \\
\hline SPT sampled & $\begin{array}{l}26(3-65 \text { years old, mean infection }= \\
2.69 \text { eggs } / 10 \mathrm{ml} \text {, range } 0-22 \text { eggs } / 10 \mathrm{ml})\end{array}$ & $\begin{array}{l}306(1.5-86 \text { years old, mean infection }= \\
0.758 \text { eggs } / 10 \mathrm{ml} \text {, range } 0-30 \text { eggs } / 10 \mathrm{ml})\end{array}$ \\
\hline
\end{tabular}

b S. haematobium infection levels by age in the selected populations

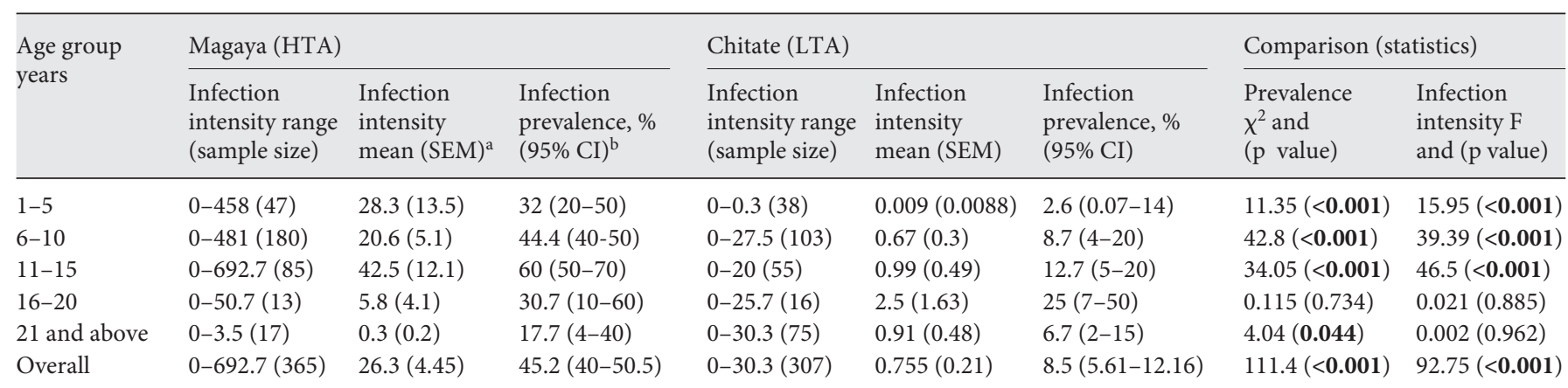

Baseline variables (a) and infection levels by age groups in the selected populations (b) are shown for the 2 villages. Statistical results of $\chi^{2}$ analysis and ANOVA on the prevalence and infection intensity, respectively, are presented for each age group and significant $\mathrm{p}$ values are highlighted in bold.

$\mathrm{CI}=$ Confidence interval; HTA $=$ high-transmission area; LTA = low-transmission area; SEM = standard error of the mean; STH $=$ soil-transmitted helminths.

\section{Antibody Profiles for Schistosome- and}

\section{Derp 1-Specific Responses}

Antischistosome adult worm IgE and IgG4 (absorbencies) were significantly higher in the high-transmission area compared to the low-transmission area (table 2). Only IgG4 responses directed against the egg stage of the parasite differed between the two villages, being also higher in the high-transmission area. Neither of the parasite-specific IgE:IgG4 ratios differed between the two areas (table 2). Anti-adult worm IgE and IgG4 responses increased with age and rose faster in the high-transmission area compared to the low-transmission area (fig. 2) and exhibited a peak shift in these responses. Amongst the Der p 1-specific responses, IgG4 levels were higher in the high-transmission area resulting in significantly lower IgE:IgG4 ratio in this village compared to the lowtransmission village (table 2).

\section{SPT Reactivity Is Related to Allergen-Specific IgE and} IgG4 Levels

Overall, the prevalence of positive SPT was 17.7\% (95\% CI 3.96-40.1) but 67.8\% (95\% CI 13.4-147.2) of SPT-positive individuals were sensitized to the HDM. Initial analyses (univariate ANOVA) confirmed that the severity of 


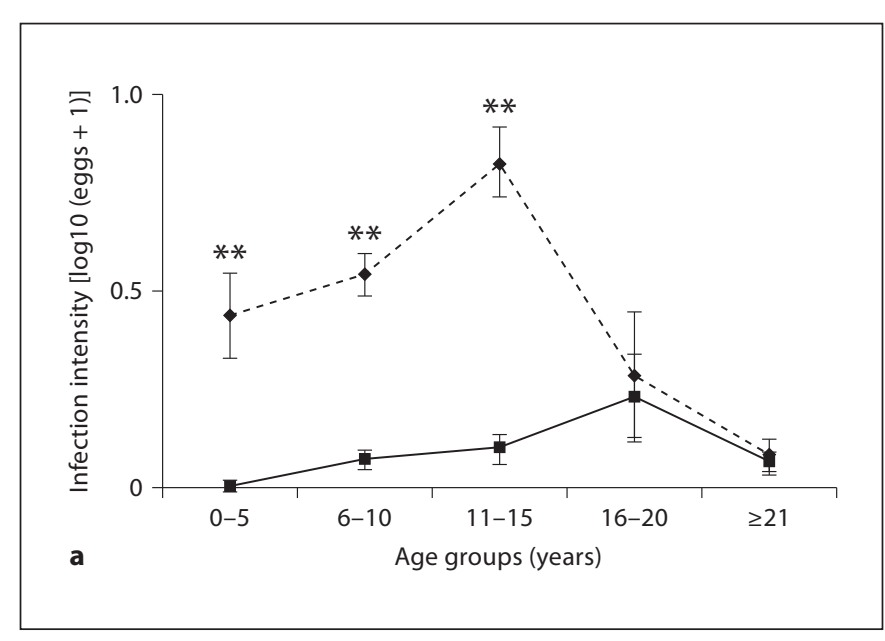

Fig. 1. Prevalence and infection intensity according to age in the study area. Infection intensity (a) and prevalence (b) are plotted against age groups in the high-transmission (-- ) and the lowtransmission area (-). Bars represent standard error of the

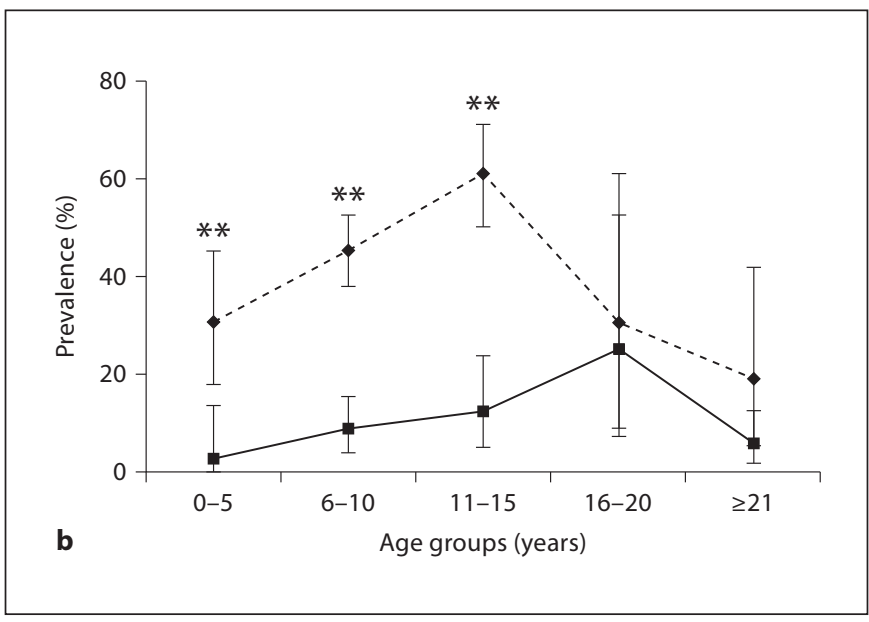

means for infection intensity and 95\% CI for the prevalence. ${ }^{*} \mathrm{p}<$ 0.05 and ${ }^{* *} \mathrm{p}<0.01$ represent significant differences in each group between the two villages.

Table 2. Results from a multivariate analysis of the effects of age, sex, village and infection intensity on antibody levels and ratio

\begin{tabular}{|c|c|c|c|c|c|c|}
\hline Dependent variable & Sex & Age & $\begin{array}{l}\text { Village } \\
\text { (comparison) }\end{array}$ & $\begin{array}{l}\text { Infection intensity } \\
F \text { and }(\beta) \text { values }\end{array}$ & $\begin{array}{l}\text { Age and village } \\
\text { interaction }\end{array}$ & $\begin{array}{l}\text { Infection and } \\
\text { village interaction }\end{array}$ \\
\hline \multicolumn{7}{|c|}{ Antischistosome responses } \\
\hline SWAP-IgE & $17.769(\mathrm{M}>\mathrm{F})^{* *}$ & $6.446^{* *}$ & $22.648(\mathrm{H}>\mathrm{L})^{* *}$ & $20.782(0.155)^{* *}$ & $3.061^{*}$ & 0.778 \\
\hline SWAP-IgG4 & $28.019(\mathrm{M}>\mathrm{F})^{* *}$ & $9.478^{* *}$ & $20.055(\mathrm{H}>\mathrm{L})^{* *}$ & $49.113(0.233)^{* *}$ & $3.54^{* *}$ & 0.139 \\
\hline SWAP-IgE/IgG4 & $2.450(\mathrm{~F}>\mathrm{M})$ & 1.895 & $1.014(\mathrm{~L}>\mathrm{H})$ & $4.948(-0.076)^{*}$ & $2.558^{*}$ & 0.001 \\
\hline SEA-IgE & $16.520(\mathrm{M}>\mathrm{F})^{* *}$ & $14.299^{* *}$ & $3.185(\mathrm{~L}>\mathrm{H})$ & $10.749(0.112)^{* *}$ & 1.804 & $5.177^{*}$ \\
\hline SEA-IgG4 & $21.685(\mathrm{M}>\mathrm{F})^{* *}$ & $15.090^{* *}$ & $42.185(\mathrm{H}>\mathrm{L})^{* *}$ & $181.472(0.416)^{* *}$ & 1.889 & $8.324^{* *}$ \\
\hline SEA-IgE/IgG4 & $0.026(\mathrm{M}>\mathrm{F})$ & 2.303 & $22.963(\mathrm{~L}>\mathrm{H})^{* *}$ & $21.026(-0.157)^{* *}$ & 1.27 & 0.096 \\
\hline \multicolumn{7}{|l|}{ Antiallergen responses } \\
\hline Der p 1-IgE & $10.769(\mathrm{~F}>\mathrm{M})^{* *}$ & $5.270^{* *}$ & $1.013(\mathrm{~L}>\mathrm{H})$ & $5.673(-0.079)^{*}$ & $3.81^{* *}$ & 0.354 \\
\hline Der p 1-IgG4 & $6.178(\mathrm{M}>\mathrm{F})^{*}$ & $4.711^{* *}$ & $15.219(\mathrm{H}>\mathrm{L})^{* *}$ & $0.527(-0.024)$ & 1.195 & 0.025 \\
\hline Der p 1-IgE/IgG4 & $11.474(\mathrm{~F}>\mathrm{M})^{*}$ & 0.806 & $11.460(\mathrm{~L}>\mathrm{H})^{* *}$ & $4.405(-0.072)^{*}$ & 1.856 & 0.853 \\
\hline
\end{tabular}

F values from a multivariate analysis determining the effects of different variables on the levels of parasite and allergen-specific antibody responses. The effects of infection intensity were determined after allowing for the effects of a host's age, sex and village of residence by sequential sums of squares. The $\beta$-coefficients from a linear regression are also shown for the direction of the relationship 'infection-antibody'. Degrees of freedom: 1 for sex, village and infection; 4 for age; the total for the error was $660 . \mathrm{F}$ values with significant $\mathrm{p}$ values (i.e. ${ }^{*} \mathrm{p}<0.05$ and ${ }^{* *} \mathrm{p}<0.01$ ) are highlighted in bold.

$\mathrm{F}=$ Female; $\mathrm{H}=$ high-transmission village; $\mathrm{L}=$ low-transmission village; $\mathrm{M}=$ male; $\mathrm{SEA}=$ soluble egg antigen; $\mathrm{SWAP}=$ soluble worm antigen preparation. the SPT reactivity to HDM was significantly associated with the levels of Der $\mathrm{p} 1 \mathrm{IgE}$ antibodies $\left(\mathrm{F}_{1,321}=37.26\right.$, $\mathrm{p}<0.001$ ), and the interaction between Der $\mathrm{p} 1$-specific IgE and IgG4 $\left(\mathrm{F}_{1,321}=5.1, \mathrm{p}=0.025\right)$. Furthermore, SPTpositive people had significantly higher Der p 1-IgE and slightly higher Der p 1-IgG4 antibody titers (absorbencies) compared to SPT-negative people (fig. 3), as determined by stepwise logistic regression analysis (Wald $\chi^{2}=11.484$, d.f. $=1, p=0.001$ and Wald $\chi^{2}=1.573$, d.f. $=1, p=0.21$, respectively, for IgE and IgG4 mean absorbencies). 


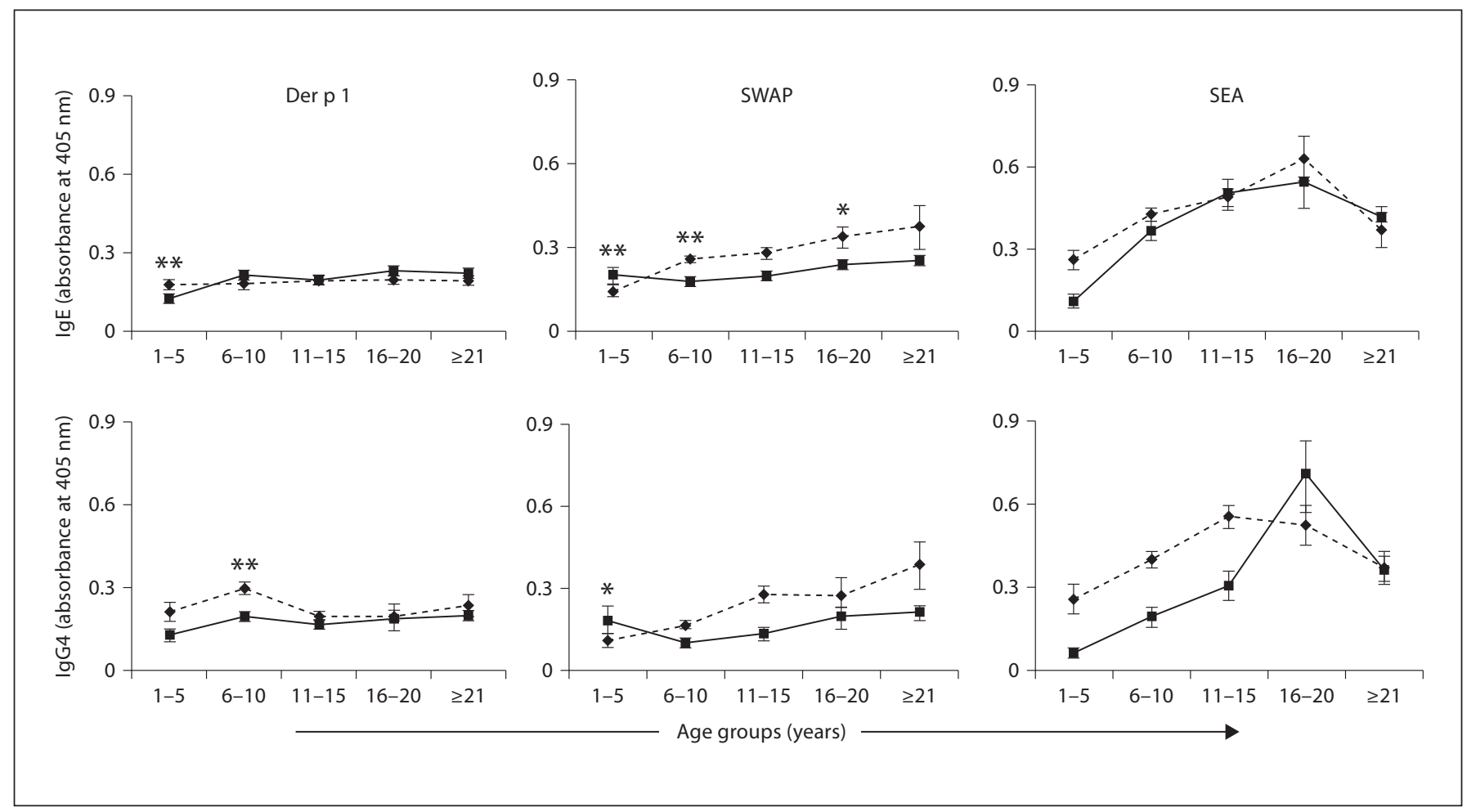

Fig. 2. Antibody levels according to age. Der p 1-specific, SWAP-specific and SEA-specific responses in the high-transmission $(---)$ and the low-transmission area (-). Bars represent standard error of the means. ${ }^{*} \mathrm{p}<0.05$ and ${ }^{* *} \mathrm{p}<0.01$ represent significant differences in each group between the two villages.

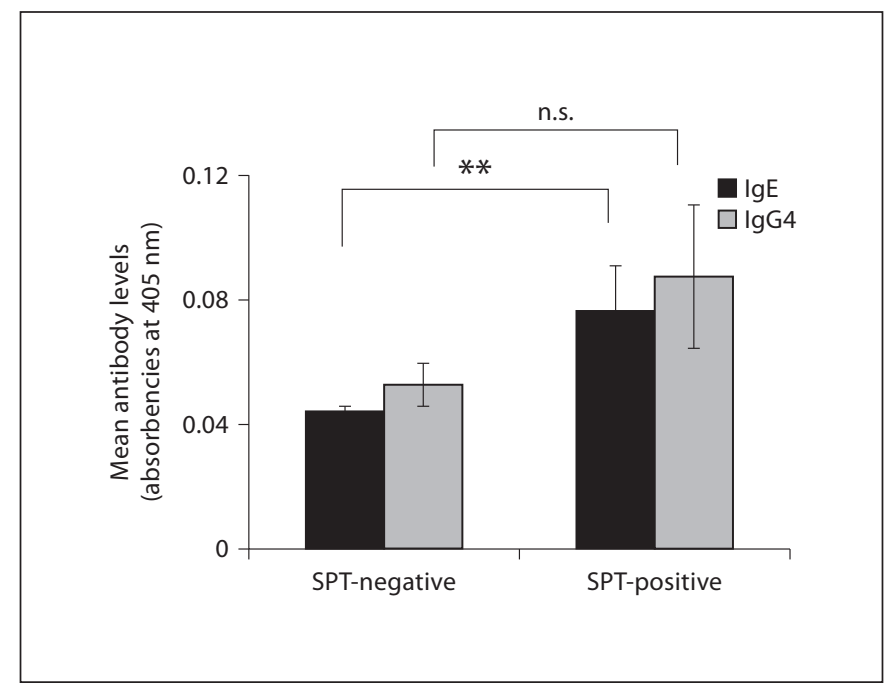

Fig. 3. Der p 1-specific antibody levels in SPT-positive versus SPTnegative individuals. Levels (absorbencies) of anti-Der p 1 IgE and IgG4 are plotted for SPT-negative and SPT-positive people. Bars represent standard error of the means. n.s. = Difference not significant, versus ${ }^{* *} \mathrm{p}<0.01=$ a significant difference.

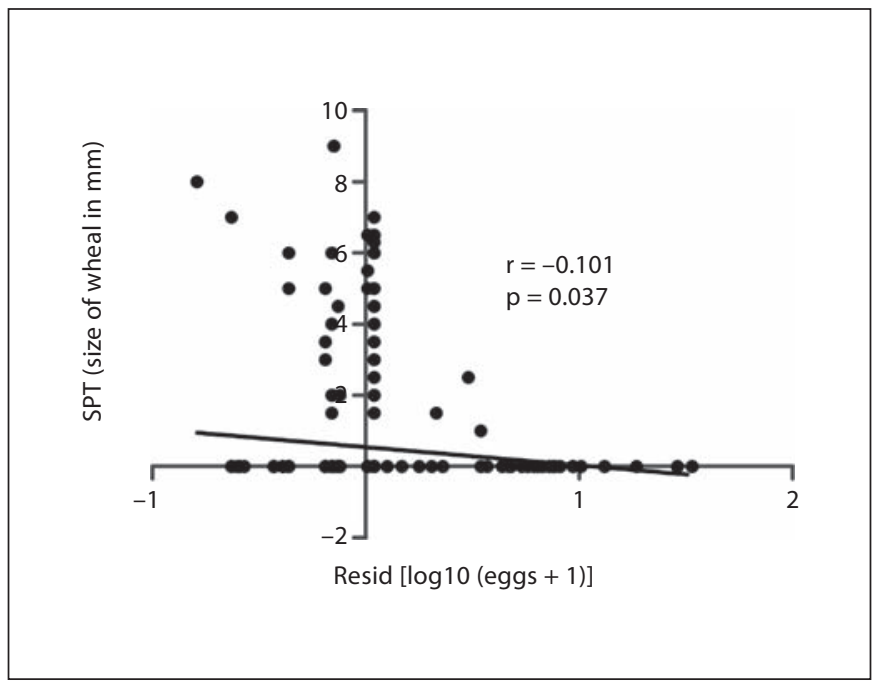

Fig. 4. Correlation between SPT results and infection intensity. Residuals of infection intensity after controlling for age, sex and village of residence are plotted against the size of the wheal in millimeters. 
Low-transmission area
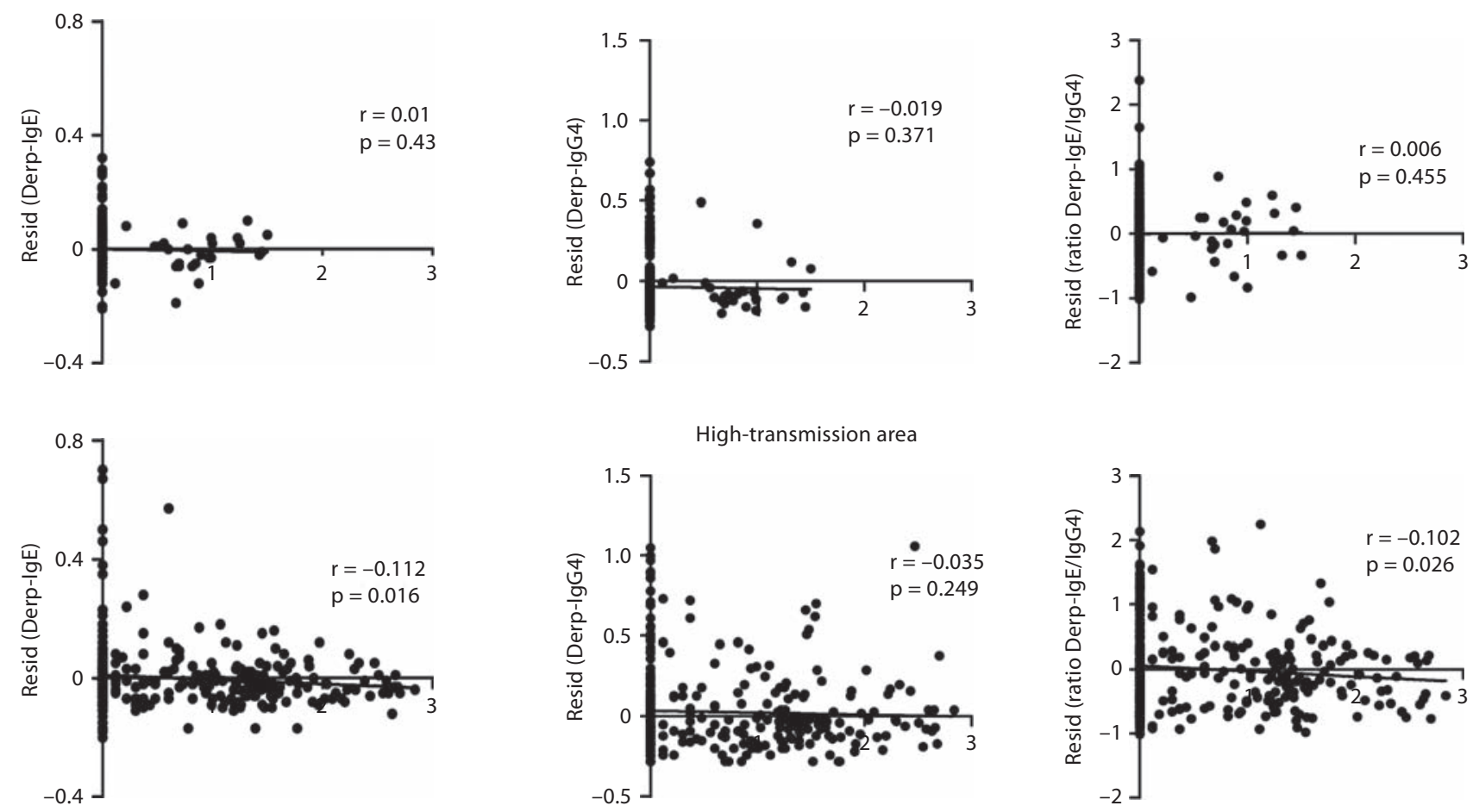

High-transmission area
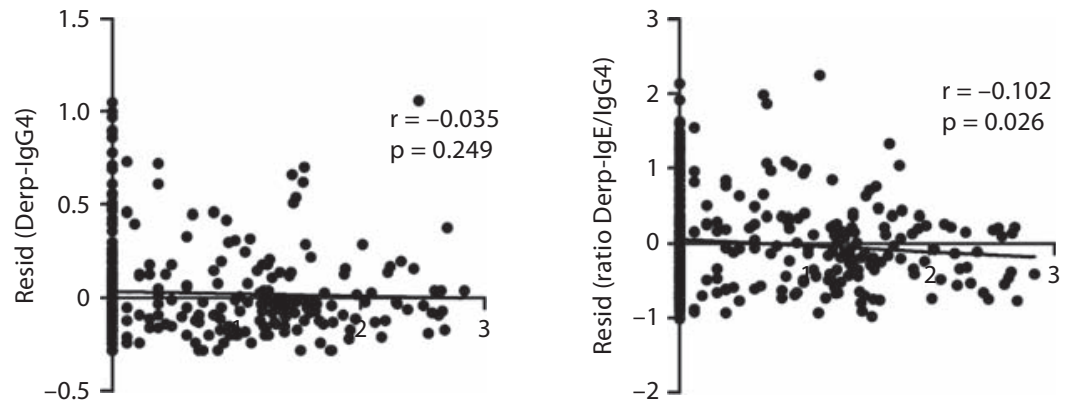

a

Infection intensity $[\log 10($ eggs +1$)]$

Low-transmission area
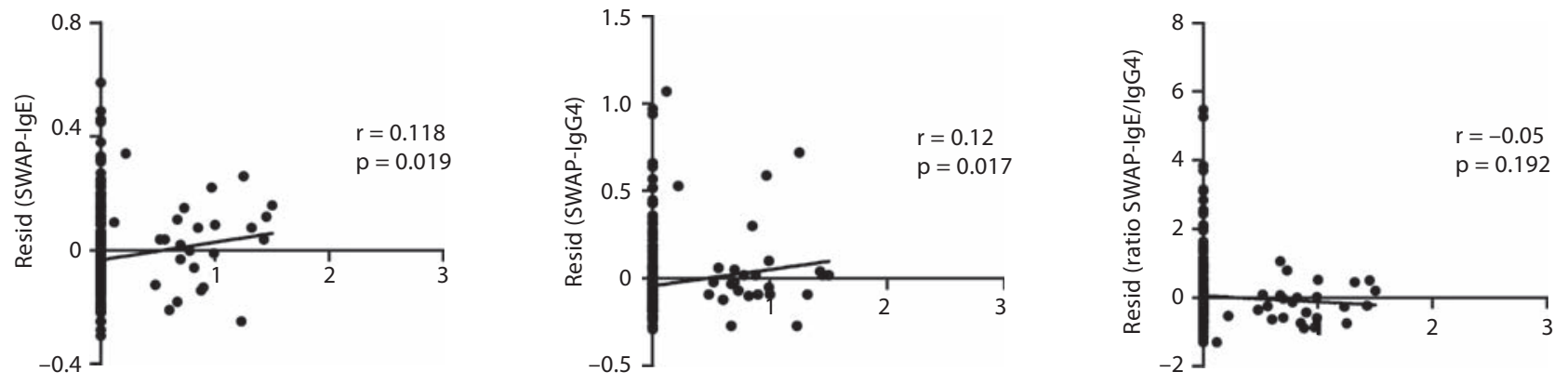

High-transmission area
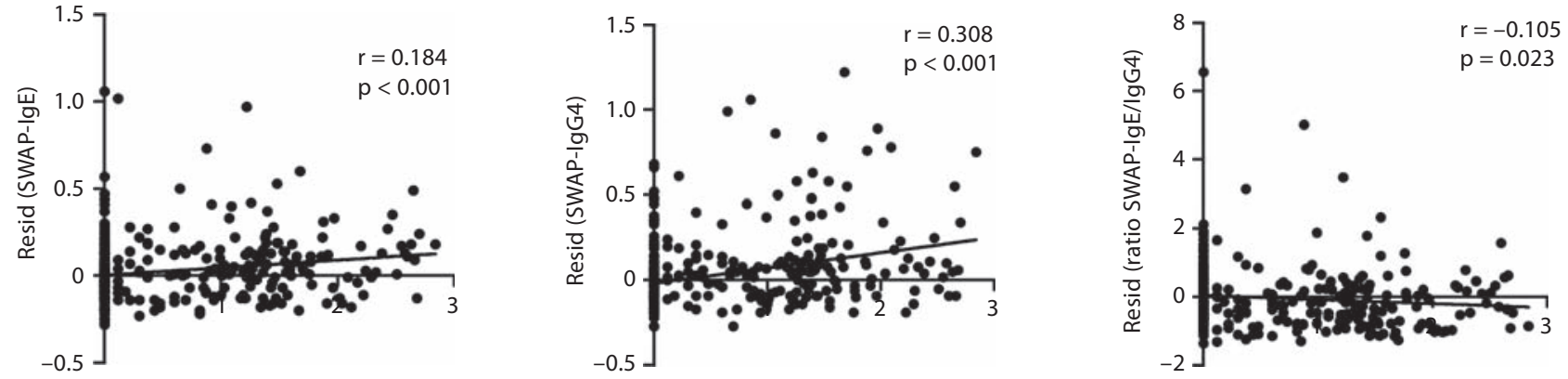

b

Infection intensity $[\log 10($ eggs +1$)]$

Rujeni/Nausch/Bourke/Midzi/Mduluza/ Taylor/Mutapi 


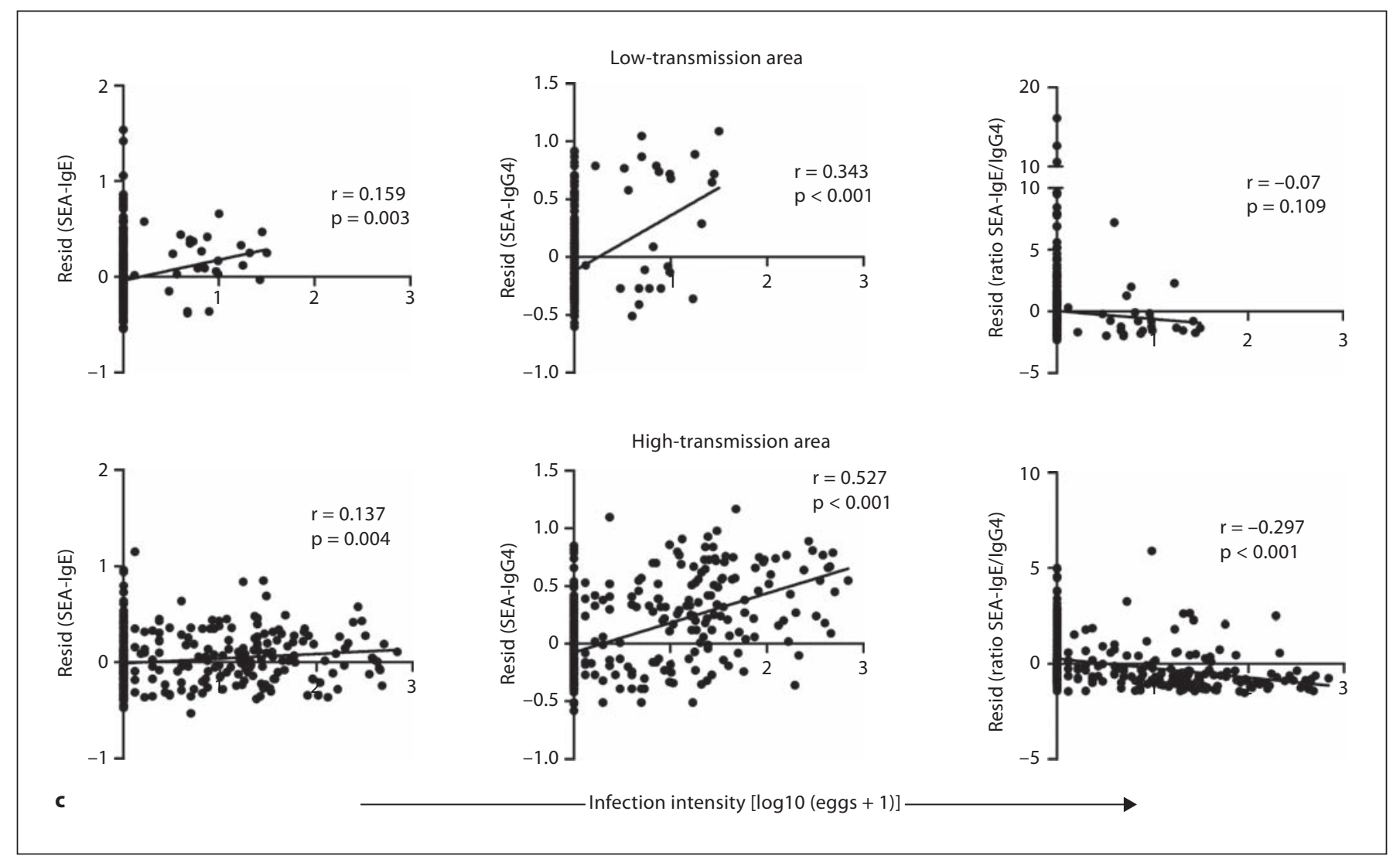

Fig. 5. Correlations between schistosome-specific:mite-specific antibody ratio and infection intensity. Residuals (after controlling for age and sex) of IgE, IgG4 and IgE/IgG4 against Der p 1 (a), SWAPs (b) and SEAs (c) are plotted against infection intensity (log-transformed) for Magaya (high-transmission area) and Chitate (low-transmission area). One-tailed Pearson correlations were performed. Correlations significant at $\mathrm{p}<0.05$ are highlighted in bold. Resid = Residual.
Atopic Responses Are Negatively Correlated with

Schistosome Infection Intensity

The prevalence of sensitization to HDM (\% of people positive for the SPT test) was higher in the low-infection area $(12.4 \%$, 95\% CI 3.48-29.1) than in the high-infection area $(7.7 \%, 95 \%$ CI $6.74-32.8)$ and SPT-negative people had higher levels of schistosome infection, though these differences were not statistically significant. However, SPT wheal size was significantly inversely related to $S$. haematobium infection intensity after allowing for the variations due to age, sex and village of residence (fig. 4). An analysis to determine factors affecting levels of Der $\mathrm{p}$ 1-specific antibodies showed that after allowing for sex, age and village of residence, schistosome infection intensity negatively affected levels (absorbencies for all individuals) of Der p 1-specific IgE, but not IgG4, resulting in a significant effect on the ratio Der p 1-IgE:IgG4 (table 2). This analysis also showed that levels of these two vari- ables differed between the two villages. Therefore, correlation analyses were conducted to determine the relationship between Der $\mathrm{p} 1$ responses (absorbencies for all individuals) and infection intensity in each of the villages (after allowing for the confounding effects of sex and age). This showed that the correlation between infection intensity and the levels of Der p 1-specific IgE and the ratio Der p 1-specific IgE:IgG4 was significant only in the high-transmission village but not in the low-transmission village (fig. 5a). This contrasted with schistosomespecific responses, where although there were differences in the magnitude of some of the responses between the two villages (table 2), the relationship between the antibodies and infection intensity was similar in the two villages (fig. 5b, c).

The analyses suggested that the lack of a relationship between schistosome infection intensity and Der $\mathrm{p} 1$ antibodies and their ratio may be due to the overall lower 
levels of infection in the low-infection area (interaction between infection and village is not significant in table 2). Therefore, the relationship between schistosome infection intensity and anti-mite antibody responses (absorbencies for all individuals) was compared in people with similar levels of infection (0-30 eggs/ $10 \mathrm{ml}$ urine) in the high-transmission village, controlling for variations due to host age and sex. This showed that at these low levels of infection, there was no significant correlation between infection intensity and Der $\mathrm{p}$ 1-specific antibodies $(\mathrm{r}=$ $-0.089, p=0.062$ for $\operatorname{IgE}$ and $r=-0.074, p=0.095$ for IgE:IgG4).

\section{Discussion}

This study compared the effect of current schistosome infection and that of the cumulative history of infection on atopic responses in two villages of different schistosome transmission dynamics. Differences in these dynamics were confirmed by the differences in schistosome infection intensity and the existence of peak shifts [29]. Moreover, we demonstrated the previously described inverse relationship between infection intensity and the ratio IgE:IgG4 against the SEA and SWAP in both villages. This ratio has been associated with the development of protective immunity against schistosome infection/reinfection $[30,31]$. In this study, the relationship was significant in the high but not in the low-transmission village. This supports previous comparative studies showing that protective immunity develops as a function of cumulative history of infection and will thus develop faster in areas of higher transmission compared to areas of lower transmission [19].

The majority of SPT-positive participants were sensitized to HDM compared to the other allergens (molds and grass) and the overall prevalence of the HDM sensitization (12\%) was comparable to that previously reported for Zimbabwean populations, and is consistent with documented levels of atopy from other African countries $[16,27,32]$. The low-infection area was overrepresented in the participants surveyed for the SPTs. However, by allowing for the potential confounding effects of residential village in the statistical analyses, it meant that the relationship between schistosome infection intensity and SPT status could be analyzed regardless of village of residence. Furthermore, the SPT participants had comparable infection ranges in the two villages, of $0-22$ versus $0-30$, i.e. within the mild infection range as classified by the World Health Organisation, which meant the analy- ses compared people with similar infection levels across the same infection range for the SPT versus infection intensity aspect of the study.

In this study, mite-specific IgE levels (absorbencies for all individuals) were positively correlated with the SPT reactivity to mite allergens, consistent with published reports [33]. Mean IgG4 levels (absorbencies) were higher, though not significantly so, in SPT-positive compared to SPT-negative people. The role of IgG4 in allergic conditions is still poorly understood, but the high levels of both IgE and IgG4 in the SPT-positive participants could be related to the fact that IgE-inducing allergens are also efficient IgG4 inducers [34]. SPT reactivity was negatively associated with $S$. haematobium infection as reported in the Gabon [10]. A comparison of Der p 1-specific antibody responses showed significant differences in levels of IgG4 but not IgE in the two villages together, with a significant difference in the IgE:IgG4 ratio. Nonetheless, IgE but not IgG4 was significantly inversely associated with schistosome infection intensity, and consequently, the ratio IgE:IgG4 was also inversely associated with schistosome infection. It seems as though the IgG4 response in this instance was modulating the pathological effects of the IgE, a concept which is important in allergy immunotherapy [35].

Differences were observed in the schistosome-antiDer $\mathrm{p} 1$ antibody relations between the two villages: there was no association between infection intensity and Der $p$ 1-specific IgE (and the ratio IgE:IgG4) antibody responses (absorbencies) in the low-transmission area but a significant negative association in the high-transmission area. This finding that areas of different schistosome endemicity exhibit differences in the relationship between antimite antibody responses and infection intensity may explain findings from a recent meta-analysis which showed that results on the relationship between schistosome infection and atopy were heterogeneous [36]. The fact that people in the low-infection area mounted similar antischistosome IgE and IgG4 responses (albeit at a lower level) indicates that there was no impairment in their ability to mount IgE and IgG4 responses. One potential reason for the lack of an association between their allergen-specific antibody responses (absorbencies) and schistosome infection was the overall low level of schistosome infection. To investigate this, the relationship between schistosome infection intensity and antimite antibody responses (absorbencies) was compared in people with similar infection levels in both villages. This showed that at such low levels of infection (0-30 eggs/10 $\mathrm{ml}$ of urine), there was no significant correlation between infection in- 
tensity and Der $\mathrm{p}$ 1-specific antibody responses in either village. This is consistent with findings from an experimental study relating $S$. mansoni infection and allergic responses [9]. This result also means that a cumulative experience of schistosome infection did not significantly affect antimite antibody responses since egg-negative older people (with a longer history of exposure [13]) did not differ in their antimite antibody responses when compared to younger egg-negative people with a shorter history of exposure to schistosome parasites, suggesting that immunomodulatory effects are only observed in viable worm infection [37].

To our knowledge, this study is the first comparative investigation conducted in two populations with the same ethnic group and socioeconomic status but different levels of schistosome infection, determining the effects of infection intensity on HDM sensitization. Overall, the study shows that regardless of the underlying biological mechanisms, the relationship between schistosome infection and HDM sensitization differs in areas of distinct schistosome infection levels, and that this difference is dependent on current infection levels and not on a history of infection. Furthermore, at low levels of infection, even in a high-transmission area, there was no relationship between infection intensity and antimite antibody responses.

Understanding the relationship between helminth infection and atopy at different infection intensities, as well as in areas with different transmission dynamics, is important not only for evaluating the long-term consequences of the current large-scale global antihelminthic treatment programs but may also influence the management of atopic diseases in people with concomitant helminth infections. In Zimbabwe, the majority of people showing the clinical signs of allergy, i.e. allergic rhinitis, conjunctivitis, asthma, dermatitis and urticaria, are sen- sitized to the HDM (measured by SPT or allergen-specific IgE) [16]. Nonetheless, differences in the relationship between helminth infection and skin sensitization compared to those between helminth infection and allergic disease [38] have been reported. Thus, further studies on the role of different parasite transmission dynamics in the clinical manifestation of atopy are required to inform therapeutic interventions [39] and the management of clinical allergy in helminth-endemic areas. Finally, the heterogeneities in the relationship between different helminth species and atopy also need to be studied in the light of the evidence that the effects of nematodes may vary from those of trematodes and between nematode species [36].

\section{Acknowledgements}

We are very grateful for the cooperation of the Ministry of Health and Child Welfare in Zimbabwe, the Provincial Medical Director of Mashonaland East, the environmental health workers and nursing staff at Chitate and Chitowa Clinics and Murehwa Hospital and residents, teachers and schoolchildren in Magaya, Chitate and Chipinda Schools. We thank members of the National Institutes of Health Research in Zimbabwe and the Biochemistry Department at the University of Zimbabwe for technical support. We also thank Susan Brown for the training on SPTs, and Jürgen Schwarze and Rick Maizels for comments on the manuscript.

The study received funding from the World Health Organization (Grant No. RPC264), The Welcome Trust (Grant No. WT082028MA), the University of Edinburgh and the government of Rwanda.

\section{Disclosure Statement}

The authors declare no financial or personal conflict of interest which could interfere with the study outcome.

\section{References}

1 Johansson SG, Bieber T, Dahl R, Friedmann PS, Lanier BQ, Lockey RF, Motala C, Ortega Martell JA, Platts-Mills TA, Ring J, Thien F, Van Cauwenberge P, Williams HC: Revised nomenclature for allergy for global use: report of the Nomenclature Review Committee of the World Allergy Organization, 2003. J Allergy Clin Immunol 2004;113: 832-836.

- 2 Flohr C, Weiland SK, Weinmayr G, Bjorksten B, Braback L, Brunekreef B, Buchele G, Clausen $\mathrm{M}$, Cookson WO, von Mutius E,
Strachan DP, Williams HC: The role of atopic sensitization in flexural eczema: findings from the International Study of Asthma and Allergies in Childhood phase 2. J Allergy Clin Immunol 2008;121:141-147.

3 Cooper PJ: Can intestinal helminth infections (geohelminths) affect the development and expression of asthma and allergic disease? Clin Exp Immunol 2002;128:398404.

4 Wills-Karp M, Santeliz J, Karp CL: The germless theory of allergic disease: revisiting the hygiene hypothesis. Nat Rev Immunol 2001;1:69-75.

5 Worldwide variation in prevalence of symptoms of asthma, allergic rhinoconjunctivitis, and atopic eczema: ISAAC. The International Study of Asthma and Allergies in Childhood Steering Committee. Lancet 1998;351: 1225-1232.

6 Maizels RM, Yazdanbakhsh M: Immune regulation by helminth parasites: cellular and molecular mechanisms. Nat Rev Immunol 2003;3:733-744. 
7 Palmer LJ, Celedon JC, Weiss ST, Wang B, Fang Z, Xu X: Ascaris lumbricoides infection is associated with increased risk of childhood asthma and atopy in rural China. Am J Respir Crit Care Med 2002;165:14891493.

-8 Lynch NR, Palenque M, Hagel I, DiPrisco MC: Clinical improvement of asthma after antihelminthic treatment in a tropical situation. Am J Respir Crit Care Med 1997;156: 50-54.

$\checkmark 9$ Smits HH, Hammad H, van Nimwegen M, Soullie T, Willart MA, Lievers E, Kadouch J, Kool M, Kos-van Oosterhoud J, Deelder AM, Lambrecht BN, Yazdanbakhsh M: Protective effect of Schistosoma mansoni infection on allergic airway inflammation depends on the intensity and chronicity of infection. J Allergy Clin Immunol 2007;120: 932-940.

10 van den Biggelaar AH, van Ree R, Rodrigues LC, Lell B, Deelder AM, Kremsner PG, Yazdanbakhsh M: Decreased atopy in children infected with Schistosoma haematobium: a role for parasite-induced interleukin-10. Lancet 2000;356:1723-1727.

-11 Wilson MS, Taylor MD, Balic A, Finney CA, Lamb JR, Maizels RM: Suppression of allergic airway inflammation by helminth-induced regulatory T cells. J Exp Med 2005, 202:1199-1212.

12 Cooper PJ, Barreto ML, Rodrigues LC: Human allergy and geohelminth infections: a review of the literature and a proposed conceptual model to guide the investigation of possible causal associations. Br Med Bull 2006;79-80:203-218.

-13 Mutapi F, Ndhlovu PD, Hagan P, Woolhouse ME: A comparison of humoral responses to Schistosoma haematobium in areas with low and high levels of infection. Parasite Immunol 1997;19:255-263.

- 14 Woolhouse MEJ, Taylor P, Matanhire D, Chandiwana SK: Acquired immunity and epidemiology of Schistosoma haematobium. Nature 1991;351:757-759.

15 Kambarami RA, Marechera F, Sibanda EN, Chitiyo ME: Aero-allergen sensitisation patterns amongst atopic Zimbabwean children. Cent Afr J Med 1999;45:144-147.

-16 Sibanda EN: Inhalant allergies in Zimbabwe: a common problem. Int Arch Allergy Immunol 2003;130:2-9.
Fulford ACJ, Butterworth AE, Sturrock RF, Ouma JH: On the use of age intensity data to detect immunity to parasitic infections, with special reference to Schistosoma mansoni in Kenya. Parasitology 1992;105:219-227.

18 Mutapi F, Hagan P, Ndhlovu P, Woolhouse MEJ: Comparison of humoral responses to Schistosoma haematobium in areas with high and low levels of infection. Parasite Immunol 1997; 19:255-263.

19 Woolhouse MEJ, Hagan P: Seeking the ghost of worms past. Nat Med 1999;5:1225-1227.

20 Blackwell AD, Gurven MD, Sugiyama LS, Madimenos FC, Liebert MA, Martin MA, Kaplan HS, Snodgrass JJ: Evidence for a peak shift in a humoral response to helminths: age profiles of IgE in the Shuar of Ecuador, the Tsimane of Bolivia, and the US NHANES. PLoS Negl Trop Dis 2011;5:e1218.

21 Midzi N, Mtapuri-Zinyowera S, Mapingure MP, Sangweme D, Chirehwa MT, Brouwer KC, Mudzori J, Hlerema G, Mutapi F, Kumar N, Mduluza T: Consequences of polyparasitism on anaemia among primary school children in Zimbabwe. Acta Trop 2010;115:103111.

22 Taylor P, Makura O: Prevalence and distribution of schistosomiasis in Zimbabwe. Ann Trop Med Parasitol 1985;79:287-299.

23 Mott KE: A reusable polyamide filter for diagnosis of S. haematobium infection by urine filtration. Bull Soc Pathol Exot Filiales 1983; 76:101-104

24 Katz N, Chaves A, Pellegrino J: A simple device for quantitative stool thick-smear technique in Schistosoma mansoni. Rev Inst Med Trop Sao Paulo 1972;14:397-400.

25 Reilly L, Magkrioti C, Mduluza T, Cavanagh DR, Mutapi F: Effect of treating Schistosoma haematobium infection on Plasmodium falciparum-specific antibody responses. BMC Infect Dis 2008;8:158.

26 Milner T, Reilly L, Nausch N, Midzi N, Mduludza T, Maizels RM, Mutapi F: Circulating cytokine levels and antibody responses to human Schistosoma haematobium: IL-5 and IL-10 levels depend upon age and infection status. Parasite Immunol 2010;32:710-721.

-27 van den Biggelaar AH, Lopuhaa C, van Ree $R$, van der Zee JS, Jans J, Hoek A, Migombet B, Borrmann S, Luckner D, Kremsner PG, Yazdanbakhsh M: The prevalence of parasite infestation and house dust mite sensitization in Gabonese schoolchildren. Int Arch Allergy Immunol 2001;126:231-238

28 Mutapi F, Roddam A: P values for pathogens: statistical inference from infectious-disease data. Lancet Infect Dis 2002;2:219-230.
29 Woolhouse ME: Patterns in parasite epidemiology: the peak shift. Parasitol Today 1998; 14:428-434.

- 30 Hagan P, Blumenthal UJ, Dunn D, Simpson AJ, Wilkins HA: Human IgE, IgG4 and resistance to reinfection with Schistosoma haematobium. Nature 1991;349:243-245.

31 Zhang Z, Wu H, Chen S, Hu L, Xie Z, Qiu Y, Su C, Cao JP, Wu Y, Zhang S, Wu G: Association between IgE antibody against soluble egg antigen and resistance to reinfection with Schistosoma japonicum. Trans R Soc Trop Med Hyg 1997;91:606-608.

32 Williams H, Robertson C, Stewart A, AitKhaled N, Anabwani G, Anderson R, Asher I, Beasley R, Bjorksten B, Burr M, Clayton T, Crane J, Ellwood P, Keil U, Lai C, Mallol J, MartinezF, Mitchell E, Montfort S, Pearce N, Shah J, Sibbald B, Strachan D, Von Mutius E, Weiland S: Worldwide variations in the prevalence of symptoms of atopic eczema in the International Study of Asthma and Allergies in Childhood. J Allergy Clin Immunol 1999; 103:125-138.

33 Busse WW, Rosenwasser LJ: Mechanisms of asthma. J Allergy Clin Immunol 2003; 111:S799-S804.

34 Aalberse RC, Stapel SO, Schuurman J, Rispens T: Immunoglobulin G4: an odd antibody. Clin Exp Allergy 2009;39:469-477.

35 Bullock RJ, Barnett D, Howden ME: Immunologic and clinical responses to parenteral immunotherapy in peanut anaphylaxis - a study using IgE and IgG4 immunoblot monitoring. Allergol Immunopathol (Madr) 2005;33:250-256.

-36 Feary J, Britton J, Leonardi-Bee J: Atopy and current intestinal parasite infection: a systematic review and meta-analysis. Allergy 2011;66:569-578.

37 Osada Y, Kanazawa T: Parasitic helminths: new weapons against immunological disorders. J Biomed Biotechnol 2010;2010:743758.

38 Flohr C, Tuyen LN, Quinnell RJ, Lewis S, Minh TT, Campbell J, Simmons C, Telford G, Brown A, Hien TT, Farrar J, Williams H, Pritchard DI, Britton J: Reduced helminth burden increases allergen skin sensitization but not clinical allergy: a randomized, double-blind, placebo-controlled trial in Vietnam. Clin Exp Allergy 2010;40:131-142.

39 Harnett W, Harnett MM: Helminth-derived immunomodulators: can understanding the worm produce the pill? Nat Rev Immunol 2010;10:278-284. 